\title{
Aseptic Spondylodiscitis Resulting from Intradiscal Radiofrequency Ablation (IDRA) in Patients with Herniated Disc Disease: A Report of Ten Cases
}

\author{
Farid Yudoyono, ${ }^{1,2}$, Do Young Kim², Dong Kyu Chin ${ }^{3}$, Dong Ah Shin ${ }^{2}$ \\ ${ }^{1}$ Department of Neurosurgery, Hasan Sadikin Hospital, College of Medicine, Padjadjaran University Bandung, Jawa Barat, Indonesia, \\ ${ }^{2}$ Department of Neurosurgery, Spine and Spinal Cord Institute, Severance Hospital, Yonsei University College of Medicine, Seoul, Korea, \\ ${ }^{3}$ Department of Neurosurgery, Spine and Spinal Cord Research Institute, Gangnam Severance Hospital Yonsei University College of Medicine, \\ Seoul, Korea
}

Corresponding Author:

Dong Ah Shin, MD, PhD

Department of Neurosurgery, Spine and Spinal Cord Institute, Severance Hospital, Yonsei University College of Medicine, 50-1, Yonsei-ro, Seodaemun-gu, Seoul 03722, Korea

Tel: $+82-2-2228-2150$

Fax: +82-2-393-9979

E-mail: shindongah@me.com

Received: June 6, 2017 Revised: November 8, 2017 Accepted: January 3, 2018
Objective: The purpose of this study was to investigate the clinical presentation, radiological features, management, and outcomes of aseptic spondylodiscitis after Intradiscal radiofrequency ablation (IDRA). Methods: Between 2014 and 2015, ten patients were diagnosed with aseptic spondylodiscitis due to significant back pain after IDRA and were assessed with medical record review, telephone interviews, and imaging. The follow-up period was 12 months. Results: The patients developed significant axial pain after the procedure (VAS, $2 \pm 1.6$ to $8 \pm 0.7, p<0.001$ ). Laboratory findings, including WBC, ESR, and CRP, were nonspecific. Magnetic Ressonance Imaging (MRI) showed low intensity on T1-weighted images, heterogeneous intensity on T2weighted images, and contrast enhancement on the subchondral area adjacent to the endplate. All patients were treated conservatively with non-steroidal anti-inflammatory drugs. Back pain improved gradually in 3 months (VAS, $5.5 \pm 1.3, p<0.01$ ). All patients had no sequelae at final follow up. Conclusion: IDRA may cause thermal injury to adjacent vertebrae. If a patient complains of significant back pain after IDRA and show osteonecrosis characteristic on MRI, aseptic spondylodiscitis should be considered. To prevent this complication, the correct location of the IDRA tip distant from the endplate should be confirmed by fluoroscopy during the procedure.

Key Words: Magnetic Ressonance Imaging, Spondylodiscitis, Radiofrequency, Herniated intervertebral disc disease

\section{INTRODUCTION}

Intradiscal radiofrequency ablation (IDRA) is a type of percutaneous disc decompression that is associated with many advantages. It does not require general anesthesia, involves a brief hospital stay, does not lead to postoperative epidural scar formation, preserves spinal stability, and allows for subsequent conventional open disc surgery, if needed. IDRA uses electrical energy to remove the nucleus pulposus and to decompress the intradiscal pressure. Favorable treatment outcomes have been reported with low complication rates ${ }^{1,2)}$.

Aseptic spondylodiscitis is defined as spinal inflammation without infectious etiologies. The pathophysiology of this syndrome remains poorly understood. It is associated with rare conditions, such as SAPHO (synovitis, acne, pustulosis, hyperostosis, and osteomyelitis) syndrome. Patients with this condition have an aseptic lesion of the spine and/or sternoclavicular region ${ }^{5}$. Vertebral inflammation caused by thermal injury can also be diagnosed as aseptic spondylitis. Spinal involvement may manifest as osteosclerosis, osteosclerosis with increased bone size, paravertebral ossification, lesions at the disco vertebral junction, or vertebral collapse. Vertebral corner erosion is consistently visible on magnetic resonance imaging (MRI) of this syndrome ${ }^{3)}$.

While IDRA has shown favorable surgical outcomes with minimal complications, excessive heat may theoretically induce thermal injury to adjacent structures. We experienced a consecutive series of patients with aseptic spondylodiscitis due to thermal injury to the spine after IDRA. Previous studies have reported aseptic spondylodiscitis after other intradiscal ablation therapies, but, to our knowledge, our study is the first to report such after IDRA ${ }^{3,4)}$. The purpose of this study was to investigate the 
clinical presentation, radiological features, management, and outcomes of aseptic spondylodiscitis after IDRA.

\section{MATERIALS AND METHODS}

\section{Patients}

Ten patients were referred to our clinic from 2014 to 2015 for increased back pain after IDRA and a presumed diagnosis of postprocedural spondylodiscitis whereas it contain three cervical and seven lumbar discs. The medical records and radiological images were reviewed retrospectively. Postoperative MRI was obtained within one week after the procedure in all patients due to significant pain. Patients were examined with a $1.5 \mathrm{~T}$ system (GE Healthcare Korea, Seoul, Republic of Korea). MRI included T1-weighted and T2-weighted sagittal and axial images. The following protocol was used: sagittal spin-echo T1W images (TR/TE: 500-640/11 ms), fast spin-echo T2W images (TR/TE: 4,000-5,000/90-102 ms), and axial spin-echo T1W images (TR/TE: 500-700/11-15 ms). The T1W sequences were repeated within 15 min of contrast-medium injection Magnevist, Schering, Nycomed, New Jersey, USA). The slice thickness was $5 \mathrm{~mm}$, with $1-\mathrm{mm}$ intervals in both planes. The signal intensity of the bone marrow was compared with that of the adjacent vertebrae. Signal intensity was assessed as low, iso or high intensity on T1W, T2W, and contrast-enhanced images. Clinical evaluation was performed by D.K.C and D.A.S. Patients were asked to complete their neck and low back the Oswestry Disability Index (ODI) and grade pain intensity using a subjective visual analog scale at routine postoperative intervals of $1,3,6$, and 12 months. The ODI scores varied from 0 to 100 (0, no disability; 100, maximum disability). Pain intensity was reported from 0 to 10 (VAS: 0 , no pain; 10, worst pain imaginable).

\section{Intradiscal RF Ablation}

A physician performed IDRA for all patients as follows. A prophylactic intravenous antibiotic was administered 30 minutes before the procedure. Patients were monitored with electrocardiography, pulse oximetry, and for blood pressure. Sedatives were administered as necessary during anesthetization of the skin and subcutaneous fascia.

Patients were placed in a prone position on the surgical table. A standard posterior lateral approach to the intervertebral disc was used. A 25-gauge needle was first inserted into the ipsilateral side of the target disc nucleus, and $1 \mathrm{~mL}$ to $2 \mathrm{~mL}$ of contrast was injected to outline the disc herniation. The skin and subcutaneous tissue were infiltrated with 1\% lidocaine. A 16-gauge introducer needle, which was manually curved, was advanced toward the lateral edge of the superior articular process. After the lateral edge was touched, the needle was rotated to point toward the midline. As the needle tip was directed toward the midline, the antero-posterior (AP) projection was checked regularly to assure the needle tip was following the correct path. Herniation entry was identified by a sudden loss of resistance.
After confirming the introducer needle position with the lateral and AP views, the wand was advanced to the center of the lesion under fluoroscopic monitoring (L'Disq, U\&I, Republic of Korea) ${ }^{8)}$. The entire procedure was monitored and evaluated with C-arm fluoroscopy.

\section{Statistical Analysis}

The paired t-test was used to compare VAS in the pre and post procedures. Statistical analysis was performed using SPSS software package version 10 (SPSS Inc., Chicago, IL, USA), and all values were expressed as mean \pm standard deviation (SD) and defined as statistically significant in the case of $p<0.05$.

\section{RESULTS}

\section{Patient Profiles}

Patient age at the time of the procedure ranged from 32 years to 60 years (mean, 50.2 years). The male to female ratio was 7:3. All patients were observed clinically for until 1 year. All patients had a history of radicular pain refractory to conservative treatments for a minimum of 3 months. The treated spinal levels included 3 cervical and 7 lumbar vertebrae. There were no laboratory examination abnormal values. On admission to our clinic, laboratory examination was repeated, including WBC, ESR, and CRP. However, all values were within normal limits.

\section{Clinical Manifestations}

All patients underwent IDRA for radicular pain, rather than axial pain. Pain was successfully controlled with IDRA (VAS immediate post op, $8 \pm 1.6$ to VAS 12 months $0.9 \pm 1, p<0.001$ ). However, the patients developed significant axial pain early after the procedure (VAS pre op, $2 \pm 1.6$ to VAS immediate $8 \pm 0.7, p<0.001$ ), and the pain persisted signficantly until 3 months postoperatively (Fig. 1). Although the initial presenting symptom was controlled

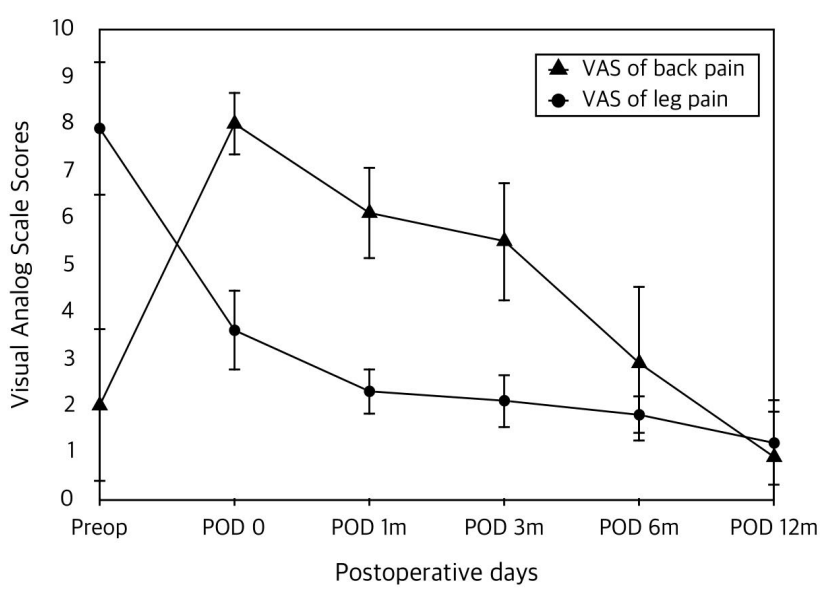

Fig. 1. Visual Analogue Score. 
Table 1. Patient demographic and initial MRI finding of aseptic spondylodiscitis

\begin{tabular}{|c|c|c|c|c|c|c|c|c|c|c|c|c|}
\hline \multirow{2}{*}{ No } & \multirow{2}{*}{ Level } & \multirow{2}{*}{ Age } & \multirow{2}{*}{ Sex } & \multirow{2}{*}{ WBC } & \multirow{2}{*}{ ESR } & \multirow{2}{*}{ CRP } & \multicolumn{6}{|c|}{ Visual Analog Scale (VAS) } \\
\hline & & & & & & & Pre & Immediate & $1 \mathrm{mo}$ & $3 \mathrm{mo}$ & $6 \mathrm{mo}$ & $12 \mathrm{mo}$ \\
\hline 1 & Cervical & 48 & Male & 5,000 & 20 & 4.7 & 3 & 8 & 7 & 6 & 3 & 1 \\
\hline 2 & Cervical & 52 & Male & 5,170 & 14 & 5.6 & 4 & 8 & 7 & 7 & 5 & 1 \\
\hline 3 & Lumbar & 39 & Male & 6,230 & 8 & 3.2 & 3 & 7 & 6 & 5 & 4 & 2 \\
\hline 4 & Lumbar & 58 & Female & 4,580 & 6 & 0.2 & 0 & 7 & 6 & 6 & 2 & 3 \\
\hline 5 & Lumbar & 47 & Male & 4,907 & 15 & 0.3 & 0 & 9 & 4 & 3 & 1 & 1 \\
\hline 6 & Lumbar & 53 & Female & 5,120 & 21 & 0.7 & 2 & 8 & 6 & 5 & 0 & 0 \\
\hline 7 & Cervical & 54 & Female & 4,999 & 22 & 1.2 & 3 & 8 & 5 & 4 & 4 & 0 \\
\hline 8 & Lumbar & 60 & Male & 5,931 & 10 & 1.9 & 0 & 9 & 7 & 6 & 3 & 0 \\
\hline 9 & Lumbar & 59 & Male & 6,212 & 3 & 7.4 & 1 & 8 & 7 & 7 & 5 & 1 \\
\hline 10 & Lumbar & 32 & Male & 6,354 & 2 & 4.1 & 4 & 8 & 6 & 6 & 2 & 0 \\
\hline
\end{tabular}

effectively by IDRA, the ODI scores showed significant disablity until POD 3 months due to aggravated axial pain. The referal diagnosis was septic spondylitis in most cases due to post-procedural pain aggravation and suspected MR findings. However, no patients had fever, and all infection profiles were negative. No bacteria were found in blood cultures.

\section{MR findings}

MR findings are summarized in Table 1. The results of MR examinations were not completely typical of post-surgical spondylitis. There was no abnormal signal intensity in the intervertebral disc and no marked contrast enhancement.

The signal intensity of the intervertebral disc was homogenous on T1W, T2W, and contrast-enhanced images. The following MR imaging showed subchondral edema adjacent to the site of intradiscal ablation. MR imaging showed low signal intensity on Tl-weighted images and high or low signal intensity on T2weighted images with moderate contrast-enhancement. MRI showed a round-shaped, low signal intensity on Tl-weighted images, heterogeneous signal intensity on T2-weighted images, and contrast-enhancement on the subchondral area adjacent to the endplate. Erosions of the endplates were detected on sagittal images (Figs. 2 and 3).

\section{Treatment Outcome}

All patients were treated conservatively with non-steroidal anti-inflammatory drugs.

Neck and Low back pain improved gradually and was reduced by more than half after POD 3 months. At postoperative 12 months, all patients had recovered with no sequelae.

\section{DISCUSSION}

Post-procedural spondilodiscitis is on the rise and currently represents about $30 \%$ of pyogenic spondylitis, 5 . The presenting symptoms of post-procedural spondylodiscitis include relief of initial symptoms with a return of symptoms one week to four
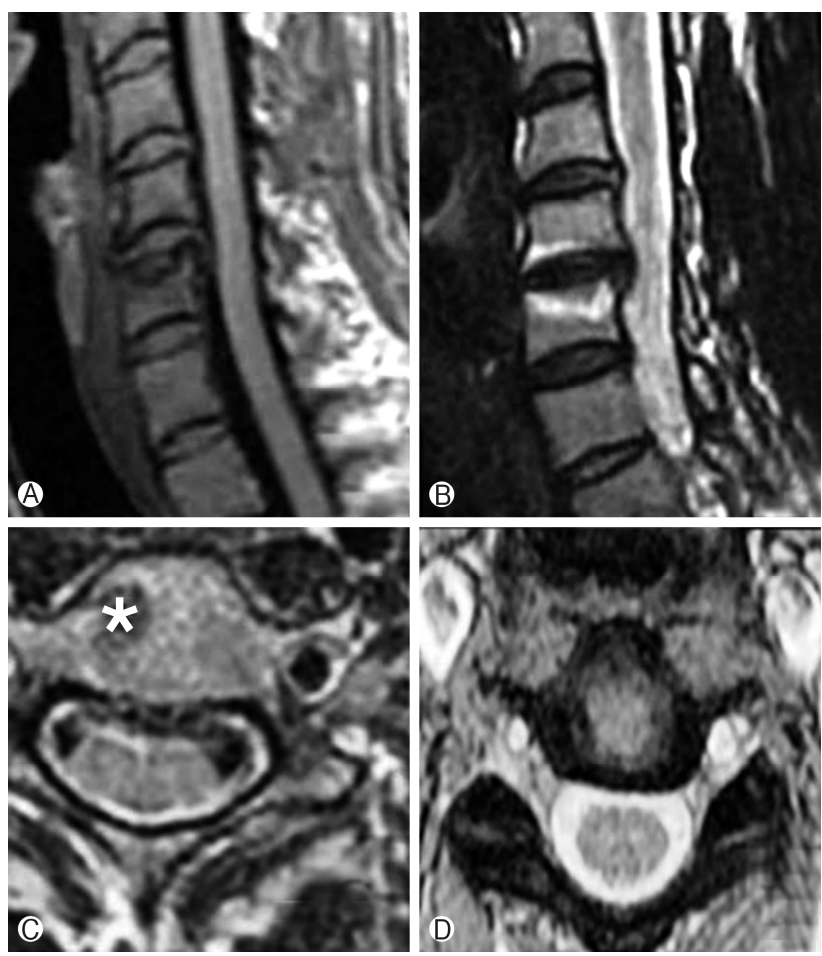

Fig. 2. A 48-year-old man developed significant neck pain 2 days after intradiscal RF ablation. MR imaging was performed 1 week later. (A) The sagittal T1-weighted image showed low signal intensity in the adjacent bone marrow. There was bone erosion in the upper endplate of C5. The C5-6 intervertebral disc showed the same signal intensity as the adjacent intervertebral discs. (B) The sagittal T2-weighted image revealed high signal intensity in the adjacent subchondral bone marrow. (C) The adjacent bone marrow showed miminal enhancement in the post-contrast T1-weighted image (asterix).

weeks postoperatively ${ }^{9)}$. However, our patients complained of aggravated neck and low back pain, regardless of leg pain, immediately after the procedure. Although fever is frequently observed in septic spondylitis, none of our patients experienced 


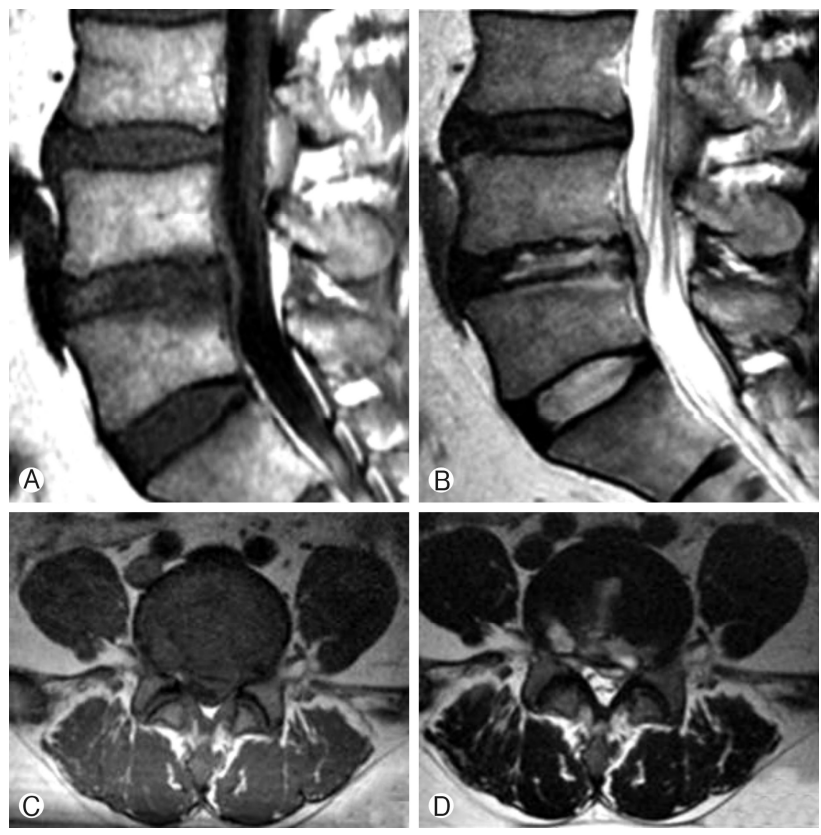

Fig. 3. A 60-year-old man developed significant back pain 2 days after intradiscal RF ablation. MR imaging was performed 5 days later. (A) The sagittal T1-weighted image showed iso to high signal intensity in the bone marrow exactly adjacent to the treated area. The L4-5 intervertebral disc showed lower signal intensity than the adjacent intervertebral discs. (B) The sagittal T2-weighted image revealed iso signal intensity in the upper adjacent bone marrow and high signal intensity in the lower adjacent bone marrow. Bone erosion was noted at the upper endplate of L5. (C) The axial T1-weighted image showed iso to high signal intensity (D). The axial T2-weighted image showed iso to high signal intensity in the intervertebral disc.

fever. In addition, none of them showed evidence of infection, as infection profiles, including WBC, and ESR were all within the normal range. In cases of borderline blood profile the authors recommend to repeat CRP examination.

We found that MR imaging produced similar characteristics to those of subchondral osteonecrosis ${ }^{7)}$. MR imaging showed high signal intensity on T2WI and low signal intensity on T1WI without enhancement adjacent to the IDRA ablation site. High T1WI and low T1WI might be caused by increased free protons in edematous necrotic tissue and reparative granulation tissue $^{12,13)}$.

The rim likely represented an advancing margin of reparative fibrous tissue with new bone formation. On the basis of these findings, we diagnosed our patients with aseptic spondylodiscitis. In experimental studies correlating MR signal intensity abnormalities of osteonecrosis with histologic examination, reduced signal intensity on T1-weighted images was attributed to necrosis of the high-signal-intensity marrow fat and replacement with an inflammatory fibrocystic infiltrate ${ }^{14,15)}$.

MRI alone is not reliable as sole method, should confirmed by CRP and disc puncture culture ${ }^{14)}$ but the authors make obser- vation for patients with high CRP due to non invasiveness.

Treatment options for this syndrome include NSAIDs, antirheumatic drugs, and corticosteroids ${ }^{5}$. Among all treatments in this recent study, NSAIDS were generally considered the firstline treatment.

The precise mechanism producing vertebral inflammation and aseptic spondylodiscitis, in our patients is unclear, although there are several possibilities. Excessive heat at the endplate may have caused thermal injury to the subchondral bone. Previous reports have shown that direct contact with articular cartilage may contribute to the development of osteonecrosis ${ }^{14,15)}$. This condition may occur when the tip of the navigable device is positioned near the endplate. All of our patients were treated with the same device. The tip of the navigable device was elastic and could be bent, so the tip might migrate to a different position when surrounding structures were ablated and removed. The heat produced from electrical fields can cause ionic agitation and friction, which then results in protein denaturing, cellular membrane disruptions, increased membrane permeability, and finally, tissue necrosis or lysis. Venous thromboembolism and embolic infarction have been suggested in the development of aseptic spondylodiscitis. Animal studies have yielded similar results showing loss of cellularity and osteonecrosis of subchondral bone ${ }^{16)}$. Our patients' lesions were larger than the thermally injured area. The lesions may have grown due to embolization of the arterioles supplying the endplate.

The main limitations of this study were the small sample size and the lack of tissue biopsy and there was no comparable placebo-treated group.

\section{CONCLUSION}

IDRA may cause thermal injury to the adjacent vertebrae. If a patient complains of severe back pain after IDRA and show osteonecrosis characteristic on MRI, aseptic spondylodiscitis should be suspected. The correct location of the RF tip should be confirmed by fluoroscopy during the procedure.

\section{Conflict of Interest}

Each author certifies that he or she, or a member of his or her immediate family, has no commercial association (i.e., consultancies, stock ownership, equity interest, patent/licensing arrangements, etc.) that might pose a conflict of interest in connection with the submitted manuscript.

\section{ACKNOWLEDGEMENT}

We acknowledge the assistance of colleagues with the Spine and Spinal Cord Institute Departement of Neurosurgery at Yonsei University Medical College, particularly Professor Dong Kyu Chin and Professor Dong Ah Shin, in making the underlying dataset available and as corresponding author. This study was supported by a grant from the Korea Health Technology R\&D Project through the Korea Health Industry Development Institute (KHIDI), 
funded by the Ministry of Health and Welfare, Republic of Korea (HC15C1320).

\section{REFERENCES}

1. Kapural L, Malak O, Arrigain S, Mekhail N: Intradiscal thermal annuloplasty versus intradiscal radiofrequency ablation for the treatment of discogenic pain: a prospective matched control trial. Pain Medicine 6:425-31, 2005

2. Fukui S, Iwashita N, Tomie H, Nosaka S, Rohof O: Results of intradiscal pulsed radiofrequency for lumbar discogenic pain: comparison with intradiscal electrothermal therapy. Korean J Pain 25:155-160, 2012

3. Cvitanic OA, Casper GD, Tirman PFJ: Subchondral marrow changes after laser diskectomy in the lumbar spine: MR imaging findings and clinical correlation. AJR 174:1363-1369, 2000

4. Tonami H, Nakagawa T, Higashi K, Okimura T, Yamamoto I, Nishijima Y: Percutaneous laser discectomy: MR findings within the first 24 hours after treatment and their relationship to clinical outcome. Clin Radiol 52:938-944, 1997

5. Kotilainen P, Saario R, Manner I, Kotilainen E: Aseptic spondylitis as the initial manifestation of the SAPHO syndrome. Eur Spine J 6:327-329, 1997

6. Gumà $M$, Pérez R, Holgado $S$, Ortiz-Santamaría V, Tena X: Aseptic spondylodiskitis in rheumatic diseases. Clin Exp Rheumatol 19:740-747, 2001

7. Tonami H, Kuginuki Y, Matoba M, Higashi HK, Yamamoto I, Nishijima V: MR imaging of subchondral osteonecrosis of the vertebral body after percutaneous laser diskectomy. AJR 173:1383-1386, 1999

8. Lee SH, Kim NH, Park HJ, Yoo HJ, Jo SY: The factors associated with the successful outcomes of percutaneous disc decompression in patients with lumbar herniated nucleus pulposus. Ann Rehabil Med 39:735-744, 2015

9. Silber JS, Vaccaro AR, Anderson PA, McCormick P: Management of postprocedural discitis. Spine J 2:279-287, 2002

10. Norman DC: Fever in the elderly. Clinical Infectious Diseases 31:148-151, 2000

11. Donowitz GR: Fever in the compromised host. Infect Disease Clin North Am 10:129-148, 1996

12. Baert AL: Magnetic Resonance Imaging in Ischaemic Stroke. New York: Springer Berlin Heidelberg pp117-118, 2006

13. Lee JD, Kim SW, Kim CS, Mun WS: MR imaging-histopathologic correlation of radiofrequency thermal ablation lesion in a rabbit liver model: observation during acute and chronic stages. Korean J Radi 2:151-158, 200114.

14. Grane P, Josephsson A, Seferlis A, Tullberg T: Septic and aseptic post-operative discitis in the lumbar spine - evaluation by MR imaging. Acta Radiologica 39:108-115, 1998

15. Janzen DL, Helms CA, Cannon D, Wright JC: Osteonecrosis after contact neodymium: yttrium aluminum garnet arthroscopic laser meniscectomy. AJR 169:855-858, 1997

16. Collier MA, Haugland LM, Bellamy J, et al: Effects of holmium: YAG laser on equine articular cartilage and subchondral bone adjacent to traumatic lesions: a histopathological assessment. Arthroscopy 9:536-545, 1993 